American Journal of Pharmaceutical Education 2019; 83 (5) Article 6970.

\title{
BRIEF
}

\section{Assessment of First-Year Student Pharmacists' Intercultural Competency Skills Using a Validated Scale and International Scenarios}

\author{
Yen Dang, PharmD, Hoai-An Truong, PharmD, MPH, Latasha Wade, PharmD \\ School of Pharmacy and Health Professions, University of Maryland Eastern Shore, Princess Anne, Maryland \\ Submitted January 22, 2018; accepted June 17, 2018; published June 2019.
}

Objective. To assess first-professional year student pharmacists' level of intercultural competency using international scenarios and a validated scale.

Methods. The Wesleyan Intercultural Competence Scale (WICS), a validated questionnaire, was administered to student pharmacists to self-assess their intercultural skills based on their responses to various situations that students encounter in international settings. Student pharmacists rated 16 items on a 5-point Likert scale ranging from "very inaccurate" to "very accurate." Their responses allowed their intercultural competency skills to be ranked among six developmental stages.

Results. The 48 participants had traveled outside of the United States an average of 2.6 times, and 34 (71\%) of them spoke another language besides English. Students' average comfort level with working with culturally diverse health care students was 4.5 out of 5 , and their average comfort level with working with patients of different cultures and background was 4.6 out of 5. The students' average overall score on the WICS was 21.9 out of 36 , which indicated the majority of the students were in the acceptance phase of cultural competency.

Conclusion. The results of this study can inform pharmacy school faculty and administrators regarding the importance of developing targeted and/or comprehensive cultural competency training for student pharmacists, in both the didactic and experiential settings, especially during global health experiences. Keywords: intercultural competency, cultural competence assessment, cultural competence training, global experiences

\section{INTRODUCTION}

Previous projections made by the US Census Bureau in 1999 estimated that the United States population would reach 394 million by $2050 .{ }^{1}$ However, based on more recent projections, the United States is expected to reach a population of more than 398 million by 2050 and exceed 416 million by 2060 , an increase of nearly $30 \%$ from the population level in $2015 .{ }^{2} \mathrm{With}$ this significant population increase, there has also been a shift in racial and ethnic diversity in the United States. Non-Hispanic whites will make up less than $50 \%$ of the US population by 2044 , and immigrants will comprise almost $20 \%$ of the total population by $2060 .^{3}$ These anticipated shifts in demographics are significant to the pharmacy profession as pharmacists will be more likely to encounter patients affected by social determinants of health, including cultural practices, beliefs, family structures, unique challenges, and health needs. To ensure new pharmacists are capable of serving

Corresponding Author: Yen Dang, School of Pharmacy and Health Professions, University of Maryland Eastern Shore, One College Backbone Rd., Princess Anne, MD 21853. Tel: 410-651-8169. E-mail: ydang@umes.edu the diverse needs of future patients, pharmacy schools must prepare and train student pharmacists with the knowledge, skills, and attitudes to deliver equitable, patient-centered care.

Cultural competency education and training are included as part of the didactic and experiential curricula in a majority of pharmacy schools in the United States, as recommended by the Center for the Advancement of Pharmaceutical Education (CAPE) 2013 Educational Outcomes and required by the Accreditation Council for Pharmacy Education (ACPE) 2016 standards. ${ }^{4-13}$ In the Doctor of Pharmacy (PharmD) curriculum at the University of Maryland Eastern Shore (UMES), cultural competency education is introduced during the first year in two required didactic courses: Concepts in Diversity and Communication for the Pharmacists ( 3 credits) and Public Health for Pharmacists ( 2 credits). Within these two required courses, student pharmacists receive nine hours of content related to diversity, health disparities, and cultural and linguistic competency. During their final year, student pharmacists complete advanced pharmacy practice experiences (APPEs) that enable them to interact with and 


\section{American Journal of Pharmaceutical Education 2019; 83 (5) Article 6970.}

provide care for diverse patient populations, including those in rural and underserved communities. Additionally, students can complete an elective APPE in public health that includes a medical mission to Haiti, Nicaragua, Peru, or Vietnam for up to two weeks.

A review of existing literature revealed that the Inventory for Assessing the Process of Cultural Competence Among Healthcare Professionals (IAPCC-R) and the Clinical Cultural Competency Questionnaire (CCCQ) have been used to assess cultural competency among student pharmacists. ${ }^{6,8,14,15}$ However, there were no studies that had employed the Wesleyan Intercultural Competence Scale (WICS) for student pharmacist assessment with respect to cultural competence. The WICS is a validated tool that measures intercultural competence based on responses to international, situational scenarios. ${ }^{16}$

The student pharmacist population enrolled in the PharmD program at UMES is diverse in many aspects, including race and ethnicity, languages abilities, and travel abroad experiences. Thus, the authors sought to examine whether student pharmacists with prior international travel would score at a higher stage of cultural competency on the WICS. We hypothesized that as student pharmacists' international travel and exposure increased, they would achieve higher developmental stages of intercultural competence on the WICS.

\section{METHODS}

The study used a cross-sectional survey. First-year student pharmacists in an accelerated, three-year PharmD program were asked to complete the WICS during the fall semester while enrolled in a required professional development course. The WICS was administered to students to self-assess their intercultural skills using a situational judgement testing approach. By this method, the questionnaire correlates situations and students' behaviors in these situations to intercultural competence levels. The WICS has been previously validated as a reliable instrument to measure intercultural competency skills. The WICS was selected for this study because it assesses behaviors, attitudes, and cognition given specific social encounters commonly experienced abroad. Participants were asked to respond to the questions in the context of recent international experiences such as study abroad or medical or global service missions as their reference. Students were given $30 \mathrm{~min}-$ utes to complete the 16-item questionnaire. The study received exemption from the university's institutional review board.

The 16-item tool assessed students' past cultural experiences in different international scenarios. Responses were based on a 5 -point Likert scale on which $1=$ very inaccurate to $5=$ very accurate. For items describing situations that students had not encountered during prior international experiences, students were instructed to check a box indicating they had not encountered that situation. These responses were not included in the data analysis. The final computed score ranked each student in one of six developmental stages of ethnocentrism and ethnorelativism. Ethnocentrism refers to judging different cultures with preconceived notions based on one's own culture, while ethnorelativism refers to the evaluation of cultures with differing values and behaviors. The first three stages (denial, defense, minimization) relate to ethnocentrism and the next three stages (acceptance, adaptation, integration) relate to ethnorelativism. The lowest stage, denial, comprises individuals who deny the existence of other cultures. The defense stage refers to individuals who criticize other cultures while promoting their own. The minimization stage refers to those who believe there are differences among cultures, but ultimately consider all cultures to be similar. Among the three higher stages of intercultural competency, acceptance refers to individuals with respect for cultural differences, adaptation refers to those who show empathy and pluralism during culturally diverse interactions, and the highest stage, integration, refers to individuals who are able to expand incorporate other worldviews into their own views.

A total WICS score was calculated and used to determine the cultural competency of each student. A detailed explanation of score calculations and distribution has been previously published. ${ }^{16}$ Statistical analyses were performed using chi-square analyses with a 95\% confidence interval. Data were analyzed using SPSS (IBM, Armonk, NY) and Minitab (Minitab, Inc., State College, PA). Permission for copyright clearance of the WICS and copies of the questionnaire were obtained by contacting the WICS corresponding author. ${ }^{16}$

\section{RESULTS}

A $100 \%(\mathrm{~N}=48)$ response rate was achieved. Demographic information for all participants is provided in Table 1 . The 48 participants had traveled an average of 2.6 times outside of the United States, and 34 (71\%) of them spoke another language besides English. Twenty (42\%) students had previously participated in cultural competency training in their workplace or the classroom prior to the completion of the WICS. Student pharmacists reported their comfort level for working with other students as 4.5 out of 5 and their comfort level for working with patients of different cultures and background as 4.6 out of 5 . 
American Journal of Pharmaceutical Education 2019; 83 (5) Article 6970.

Table 1. Baseline Demographics

\begin{tabular}{|c|c|}
\hline Characteristic & $\mathbf{N}=48$ \\
\hline \multicolumn{2}{|l|}{ Gender, $\mathrm{n}(\%)$} \\
\hline Male & $28(58.3)$ \\
\hline \multicolumn{2}{|l|}{ Race, n (\%) } \\
\hline African-American/Black & $25(52.1)$ \\
\hline Asian or Pacific Islander & $12(25.0)$ \\
\hline Age (yrs), mean (SD) & $27.6(6.3)$ \\
\hline Number of States Traveled in the USA, mean (SD) & $8.9(9.8)$ \\
\hline Number of International Countries Traveled, mean (SD) & $2.6(2.1)$ \\
\hline \multicolumn{2}{|l|}{ Distribution of International Countries, total } \\
\hline Africa & 10 \\
\hline Asia & 19 \\
\hline South America & 8 \\
\hline \multicolumn{2}{|l|}{ Ability to Speak Foreign Language, n (\%) } \\
\hline No & $14(29.2)$ \\
\hline Yes & $34(70.8)$ \\
\hline \multicolumn{2}{|l|}{ Past Cultural Competency Training, n (\%) } \\
\hline No & $28(58.3)$ \\
\hline Yes & $20(41.7)$ \\
\hline Comfort Level Working with Students with Diverse Backgrounds, mean (SD) ${ }^{\mathrm{a}}$ & $4.5(0.5)$ \\
\hline Comfort Level Working with Patients with Different Backgrounds, mean (SD) ${ }^{\mathrm{a}}$ & $4.6(0.6)$ \\
\hline
\end{tabular}

${ }^{a}$ Scoring was based on a 5-point Likert scale

Based on the WICS stages categorization, one $(2.1 \%)$ student placed in the defense phase, seven $(14.5 \%)$ students in the minimization phase, 29 (60.4\%) students in the acceptance phase, and 11 (22.9\%) students in the adaptation phase (Table 2). The average score on the WICS was 21.9 out of 36, which suggested the majority of students were in the acceptance phase of cultural competency. Students scored the lowest (average score of 0.8 ) on items describing situations when they were interacting with local peers while traveling abroad but scored highest (average of 6.9) in situations where a local peer had views that were different from their own (Table 3 ).

Table 2. Analysis of First-Year Student Pharmacists' Scores on the Wesleyan Intercultural Competency Scale

\begin{tabular}{lc}
\hline Stage & No. (\%) \\
\hline Denial & $0(0)$ \\
Defense & $1(2.1)$ \\
Minimization & $7(14.6)$ \\
Acceptance & $29(60.4)$ \\
Adaptation & $11(22.9)$ \\
Integration & $0(0)$ \\
\hline
\end{tabular}

There was no significant difference $(p=.54)$ in intercultural competence levels between students who had prior cultural competency training $(20,42 \%)$ and those who did not. There was also no significant difference $(p=.66)$ in intercultural competence levels between students who were able to speak a foreign language $(34,71 \%)$ and those who were not. Finally, there was no significant difference $(p=.18)$ in WICS scores for participants who had traveled to four or more international locations compared to those who had traveled to three or less. Gender $(p=.81)$, race $(p=.36)$, and higher self-reported scores of comfort level when working with patients of different backgrounds $(p=.53)$ were not correlated with higher WICS staging. However, those who reported higher comfort levels when working with students of diverse backgrounds were more likely to score in the acceptance and adaptation phase $(p=.04)$.

\section{DISCUSSION}

Cultural competency has been an important discussion topic in pharmacy curricula, with emphasis given to the subject in both the CAPE 2013 educational outcomes and ACPE 2016 accreditation standards. Nevertheless, 


\section{American Journal of Pharmaceutical Education 2019; 83 (5) Article 6970.}

Table 3. Score Distribution of Situations Described in the Wesleyan Intercultural Competency Scale

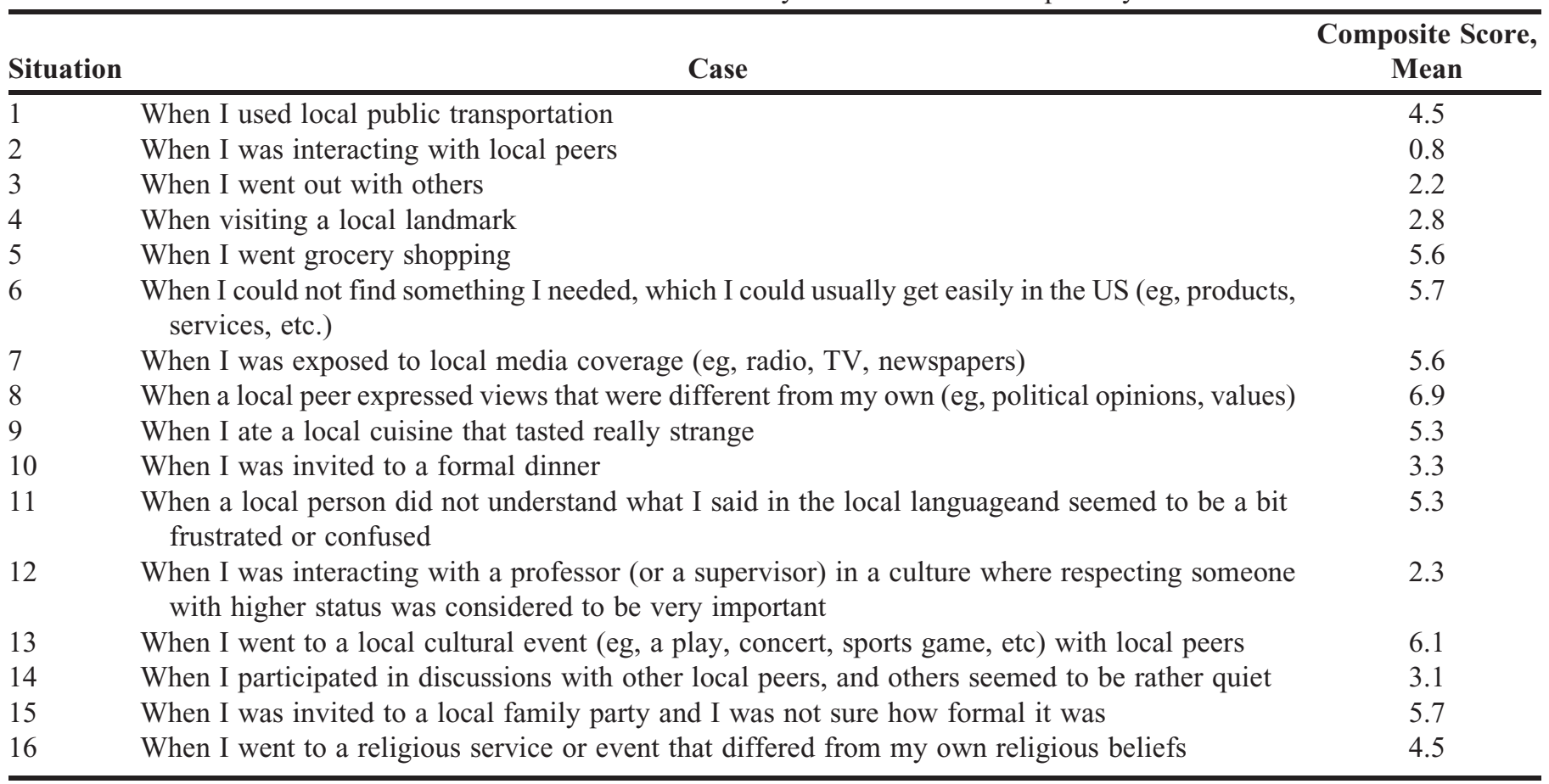

there has been limited assessment of baseline data on cultural competency among pharmacy students. The WICS is a validated and innovative tool; however, to our knowledge, it has never been used before in a study to assess cultural competency skills in health professional students.

In pharmacy and health professions education, the IAPCC-R and CCCQ are the more commonly used tools to assess cultural competency in the literature. These tests as well as the WICS are self-administered, reliable, and validated instruments for assessing cultural competency. The IAPCC-R and CCCQ were specifically designed to assess cultural competency in health care professionals and physicians, respectively, with health care delivery as the context. The WICS, however, is a situational judgement test that was designed to assess the impact that engaging in activities of daily living while participating in international experiences (eg, interaction with the native community, using local public transportation, grocery shopping, eating local cuisine, viewing local media coverage, communicating in the local language) has on the development of cultural competency. Advantages of employing the WICS in this study included the small number of questions as compared to the number of items on the IAPCC-R and CCCQ, no cost to use the assessment instrument, and ease of acquiring the assessment instrument.

The premise of this study (that as student pharmacists' international travel and exposure increased, they would achieve higher developmental stages of intercultural competence on the WICS) was not supported by our results. Nevertheless, the study addressed the literature gap related to using the WICS to assess cultural competency among student pharmacists.

The study results indicate that while most student pharmacists in their first-professional year at UMES were already in the ethnorelativism phase, there were some who needed further training and development in cultural competency. This finding validates the need for cultural competency training because these students were not culturally competent enough to care for the patient populations that they will encounter upon entering the pharmacy profession. As the students surveyed were in the first year of the pharmacy program, there was time and opportunities for their cultural competency to improve from the ethnocentrism phase to the enthnorelativism phase as they progressed through the curriculum.

Despite an increased interest in global health experiences in pharmacy education, there have been few studies published that assess cultural competency before and after student pharmacists are involved in global health experiences. ${ }^{17}$ As an extension of this study, the WICS could be readministered to the student population initially surveyed after they complete the cultural competency workshop in Public Health for Pharmacists in the second semester of their first professional year to determine whether changes in staging occurred. Additionally, this questionnaire could be administered before and after 


\section{American Journal of Pharmaceutical Education 2019; 83 (5) Article 6970.}

APPEs in which students provide care for patients and populations from diverse backgrounds, or after global experiences such as medical missions. A future research question could be whether students who achieved higher developmental stages on the WICS perform better on cultural sensitivity assessments administered during their APPEs.

The results of this study can inform faculty members seeking to develop targeted and/or comprehensive cultural competency training for student pharmacists, within both the didactic and clinical/experiential setting. This study could be replicated using cohorts from three-year accelerated programs versus traditional programs, and among other health professions students. Additionally, the results could be helpful as a hypothesis-generating study to expand further assessment, ie, compare and contrast various educational intervention and/or training of cultural competency among student pharmacists.

The financial resources and time required to complete this study were relatively nominal and included: cost to print the nine-page WICS for 48 students, one hour of faculty supervision for administration of the questionnaire, six hours for one APPE student to analyze and evaluate the results, and one hour of faculty time for reviewing and finalizing the results.

Several limitations to this study were identified. The study was not powered to detect statistical significance with the small sample size of students surveyed; therefore, the results were descriptive in nature. The inherent diversity of the sample could also be perceived as a limitation and may have biased the results as students at baseline were mostly from underrepresented groups, well-traveled globally, and multilingual, suggesting they may have already had a higher level of cultural awareness. Because of this, these results may not be applicable to pharmacy schools where the student population is not as diverse or may have had fewer international travel experiences.

Additionally, 20 (41.7\%) of the students who completed the questionnaire were already exposed to cultural competency training prior to completing the WICS. Having prior training may have heightened their perceived awareness of different cultures. These results are consistent with a previous cross-sectional study by Okoro and colleagues in which student pharmacists with prior cultural competency education and the ability to speak a foreign language reported greater comfort with addressing cultural situations. ${ }^{15}$ While formal cultural competency training is included in two required didactic courses in the UMES School of Pharmacy curriculum, students had not taken these courses before they completed the questionnaire.

Another potential limitation is that completion of the WICS is intended to be from the perspective of travel to international countries outside of the United States. However, because some respondents were born and raised outside of the United States, they may have responded to the questionnaire from the perspective of the United States being the "international" country for them. Thus, these students' responses on the WICS could have been biased and their results may not have accurately reflected their current intercultural stage.

\section{CONCLUSION}

While most of the student pharmacists in our study scored in the acceptance phase of the WICS, many did not achieve the higher stages of intercultural competence, such as adaptation and integration. Pharmacy schools should examine the content of their curriculum as well as their cocurricular offerings to enable student pharmacist exposure to topics that are global in nature. Examples of such opportunities could be the inclusion of global health topics throughout the curriculum, hosting international students, offering study abroad experiences, and facilitating faculty or instructor exchanges. This study highlights an opportunity for schools and colleges of pharmacy to further enhance their curricula with cultural competency and global health experiences. This could provide students with needed global and cultural experiences that would enhance their skills as future pharmacists in delivering patient-centered care for the changing demographics of the US population.

\section{ACKNOWLEDGMENTS}

The authors acknowledge the class of 2020 at the UMES School of Pharmacy for participating in this questionnaire.

\section{REFERENCES}

1. Wan H, Hobbs F. Minority Population Growth: 1995 to 2050, The Emerging Minority Marketplace. Washington: U.S. Department of Commerce, Minority Business Development Agency; 1999. https:// www.mbda.gov/sites/mbda.gov/files/migrated/files-attachments/ MinorityPopulationGrowth1995to2050.pdf. Accessed June 25, 2019. 2. Population Projection Program, United States Census Bureau. Table 1. Projections of the Population and Components of Change for the United States: 2015 to 2060. https://www.census.gov/data/ tables/2014/demo/popproj/2014-summary-tables.html. Accessed June 25, 2019.

3. Colby S, Ortman J. Projections of the Size and Composition of the U.S. Population: 2014 to 2060, Current Population Reports.

Washington: U.S. Census Bureau; 2014. https:/census.gov/content/ dam/Census/library/publications/2015/demo/p25-1143.pdf. Accessed June 25, 2019.

4. Assemi M, Cullander C, Hudmon KS. Implementation and evaluation of cultural competency training for pharmacy students. Ann Pharmacother. 2004;38:781-786. 


\section{American Journal of Pharmaceutical Education 2019; 83 (5) Article 6970.}

5. Evans E. An elective course in cultural competence for healthcare professionals. Am J Pharm Educ. 2006;70(3):Article 55.

6. Poirier TI, Butler LM, Devraj R, Gupchup GV, Santanello C, Lynch JC. A cultural competency course for pharmacy students. Am J Pharm Educ. 2009;73(5):Article 81.

7. Muzumdar JM, Goodman MH, Black C, Powers M. Cultural competence knowledge and confidence after classroom activities. $\mathrm{Am}$ J Pharm Educ. 2010;74(8):Article 150.

8. Haack S, Phillips C. Teaching cultural competency through a pharmacy skills and applications course series. Am J Pharm Educ. 2012;76(2):Article 27.

9. Brown B, Heaton PC, Wall A. A service-learning elective to promote enhanced understanding of civic, cultural, and social issues in health disparities in pharmacy. Am J Pharm Educ.

2007;71(1):Article 9.

10. Haack S. Engaging pharmacy students with diverse patient populations to improve cultural competence. Am J Pharm Educ. 2008;72(5):Article 124.

11. Vyas D, Caligiuri FJ. Reinforcing cultural competency concepts during introductory pharmacy practice experiences. Am J Pharm Educ. 2010;74(7):Article 129.

12. American Association of College of Pharmacy. CAPE

Educational Outcomes. http://sitefinityuc.blob.core.windows.net/
pharm-dev/docs/default-source/Program/cape-educationaloutcomes-2013.pdf?sfvrsn=fc9e7940_0. Accessed June 25, 2019. 13. Accreditation Council for Pharmacy Education (ACPE). Accreditation Standards and Key Elements for the Professional Program in Pharmacy Leading to the Doctor of Pharmacy Degree. https://www.acpe-accredit.org/pdf/Standards2016FINAL.pdf. Accessed June 25, 2019.

14. Echeverri M, Brookover C, Kennedy K. Nine constructs of cultural competence for curriculum development. Am J Pharm Educ. 2010;74(10):Article 181.

15. Okoro ON, Odedina FT, Reams RR, Smith WT. Clinical cultural competency and knowledge of health disparities among pharmacy students. Am J Pharm Educ. 2012;76(3):Article 40.

16. Stemler SE, Imada T, Sorkin C. Development and validation of the Wesleyan Intercultural Competence Scale (WICS): a tool for measuring the impact of study abroad experiences. Frontiers: The Interdisciplinary Journal of Study Abroad. 24. 25-47.

17. Gleason S, Covvey J, Abrons J. Connecting Global/International Pharmacy Education to the CAPE 2013 Outcomes: A Report from the Global Pharmacy Education Special Interest Group. American Association of College of Pharmacy White Paper. http:// www.aacp.org/resources/education/cape/Documents/ GPE_CAPE_Paper_November_2015.pdf 